Journal for ImmunoTherapy of Cancer

\title{
PODO447: a novel antibody to a tumor- restricted epitope on the cancer antigen podocalyxin
}

Diana Canals Hernaez (D , ${ }^{1}$ Michael R Hughes, ${ }^{1}$ Pamela Dean, ${ }^{2}$ Peter Bergqvist, ${ }^{3}$ Ismael Samudio, ${ }^{3}$ Ola Blixt, ${ }^{4}$ Katharina Wiedemeyer, ${ }^{5}$ Yicong Li, ${ }^{1}$ Chris Bond, ${ }^{3}$ Eric Cruz, ${ }^{3}$ Martin Köbel, ${ }^{5}$ Blake Gilks, ${ }^{6}$ Calvin D Roskelley, ${ }^{2}$ Kelly M McNagny (D) ${ }^{1}$

To cite: Canals Hernaez D, Hughes MR, Dean P, et al. POD0447: a novel antibody to a tumor-restricted epitope on the cancer antigen podocalyxin. Journal for ImmunoTherapy of Cancer 2020;8:e001128. doi:10.1136/jitc-2020-001128

- Additional material is published online only. To view, please visit the journal online (http://dx.doi.org/10.1136/jitc2020-001128).

Accepted 05 November 2020

Check for updates

(C) Author(s) (or their employer(s)) 2020. Re-use permitted under CC BY-NC. No commercial re-use. See rights and permissions. Published by BMJ.

For numbered affiliations see end of article.

Correspondence to

Professor Kelly M McNagny; kelly@brc.ubc.ca

\section{ABSTRACT}

Background The success of new targeted cancer therapies has been dependent on the identification of tumor-specific antigens. Podocalyxin (Podxl) is upregulated on tumors with high metastatic index and its presence is associated with poor outcome, thus emerging as an important prognostic and theragnostic marker in several human cancers. Moreover, in human tumor xenograft models, Podxl expression promotes tumor growth and metastasis. Although a promising target for immunotherapy, the expression of PodxI on normal vascular endothelia and kidney podocytes could hamper efforts to therapeutically target this molecule. Since pathways regulating posttranslational modifications are frequently perturbed in cancer cells, we sought to produce novel anti-Podxl antibodies (Abs) that selectively recognize tumor-restricted glycoepitopes on the extracellular mucin domain of Podxl. Methods Splenic B cells were isolated from rabbits immunized with a Podxl-expressing human tumor cell line. Abs from these $B$ cells were screened for potent reactivity to Podxl ${ }^{+}$neoplastic cell lines but not Podxl ${ }^{+}$primary endothelial cells. Transcripts encoding heavy and light chain variable regions from promising $B$ cells were cloned and expressed as recombinant proteins. Tumor specificity was assessed using primary normal tissue and an ovarian cancer tissue microarray (TMA). Mapping of the tumorrestricted epitope was performed using enzyme-treated human tumor cell lines and a glycan array.

Results One mAb (POD0447) showed strong reactivity with a variety of Podxl+ tumor cell lines but not with normal primary human tissue including Podxl+ kidney podocytes and most vascular endothelia. Screening of an ovarian carcinoma TMA (219 cases) revealed P0D0447 reactivity with the majority of tumors, including $65 \%$ of the high-grade serous histotype. Subsequent biochemical analyses determined that POD0447 reacts with a highly unusual terminal $\mathrm{N}$-acetylgalactosamine beta-1 (GaINAc $\beta 1$ ) motif predominantly found on the Podxl protein core. Finally, Ab-drug conjugates showed specific efficacy in killing tumor cells in vitro.

Conclusions We have generated a novel and exquisitely tumor-restricted mAb, POD0447, that recognizes a glycoepitope on Podxl expressed at high levels by a variety of tumors including the majority of life-threatening high-grade serous ovarian tumors. Thus, tumor-restricted P0D0447 exhibits the appropriate specificity for further development as a targeted immunotherapy.

\section{BACKGROUND}

Ovarian carcinoma (OC) is one of the leading causes of cancer mortality in women. ${ }^{1}$ Frequently diagnosed only at advanced stages and with a 5-year survival of approximately $47 \%,{ }^{2} \mathrm{OC}$ is a 'silent killer' that offers few treatment options. Although new diagnostic and treatment options have led to a significant improvement in survival rates for many cancers over the last 50 years, the 5-year survival for OC has hardly changed since $1980,{ }^{3}{ }^{4}$ highlighting the urgent need for new treatment strategies.

Although genetic and/or epigenetic changes are known to drive tumor development, dysregulation of protein expression and altered post-translational modifications are also known to occur. Specifically, changes in the patterns of protein glycosylation are well known to occur in cancer ${ }^{5}$ and, in some cases, are thought to drive tumor progression. ${ }^{6}$ This can be through well-known effects on protein stability and folding; or through modification of processes including protein trafficking, immune recognition, cell migration, ligandreceptor interaction, signal transduction and cell adhesion. ${ }^{7-11}$ Indeed, glycans can confer a selective advantage on cells that facilitates tumor spread. ${ }^{12} 13$ Moreover, cancerous cells undergo reprograming and transcriptional changes that greatly impact their glycome and glycoproteome, leading to overexpression or de novo expression of specific glycoepitopes. Importantly, these cancer-specific alterations in protein glycosylation may also provide a unique opportunity for clinical intervention with reduced toxicity since these modifications are largely tumor-specific.

Podocalyxin (Podxl) is a member of a 3-gene family of stem cell sialomucins that includes Podxl, CD34 and endoglycan. 
These proteins can be distinguished from other cell surface sialomucins based on a shared genomic organization (each encoded by a similar, 8-exon, genomic locus) and a shared protein domain structure: they each contain a large, highly glycosylated mucin domain followed by a cysteine-bonded globular domain, stalk domain, transmembrane domain and a short (approximately 70 amino acid) cytoplasmic tail with a consensus C-terminal binding site for PDZ-domain proteins. ${ }^{14-16}$ Although widely expressed during embryonic development, ${ }^{15} 17$ in normal adult tissue Podxl is primarily expressed on vascular endothelia, ${ }^{18} 19$ kidney podocytes $^{15}$ and a restricted subset of epithelial lumens. ${ }^{15}$ Nevertheless, Podxl is also aberrantly expressed by a wide variety of cancer types, and this expression is consistently an indicator of poor prognosis. ${ }^{20-30}$ For example, in early retrospective studies of breast cancer, patients with tumors expressing high levels of Podxl exhibited a greatly reduced disease-specific survival, and multivariant analysis showed Podxl to be a highlysignificant independent predictor of poor outcome. ${ }^{20}$ Similarly, in OC where Podxl is expressed by the majority of high-grade serous carcinomas, cell surface expression of Podxl correlated with a significant decrease in disease free survival. ${ }^{22}$ Interestingly, recent gene silencing experiments suggest that upregulation of Podxl is not merely a predictor of poor outcome but also an important player in disease progression by enhancing tumor invasion and metastasis. ${ }^{21} 293132$ In summary, because of its cell surface localization and its direct role in tumor metastasis, Podxl is a promising target for cancer immunotherapy.

As a first foray into exploiting this opportunity, we generated a series of anti-Podxl monoclonal antibodies (mAbs) to the extracellular domain of Podxl expressed on the surface of tumor cells. ${ }^{21}$ One mAb, PODOC1 (hereafter designated PODO83), showed a potent ability to restrict primary tumor growth in vivo and to block metastatic progression when administered to tumor bearing mice. ${ }^{21}$ While PODO83 harbors tremendous potential as a therapeutic, its ability to recognize the stalk domain of the Podxl core protein poses a possible risk of toxicity due to its reactivity with normal kidney podocytes and vascular endothelia.

Intriguingly, however, Podxl is known to undergo tissue-specific glycosylation, ${ }^{30}$ a process that is frequently altered in cancer. ${ }^{6} 3334$ Accordingly, to circumvent the potential toxicity associated with Abs reactive to the core polypeptide, we carefully rescreened our panel of anti-Podxl Abs for those that react selectively with a tumor-restricted glycoform of Podxl but not with normal tissue. Here, we report the identification of PODO447, a novel mAb with exquisite specificity for a tumor-specific glycoepitope on Podxl. As such, PODO447 offers a unique opportunity to selectively target tumor tissue while sparing normal tissue and highlights its promise as a therapeutic.

\section{METHODS}

\section{Ab production}

New Zealand White rabbits were immunized with Podxlexpressing human glioblastoma A-172 cells (\#CRL-1620, American Tissue Culture Collection (ATCG)). Rabbit mAbs were isolated using selected lymphocyte antibody method (SLAM) technology. ${ }^{35}$ Briefly, individual B-cell clones whose supernatants reacted with Podxlexpressing MDA-MB-231 cells were selected. These were then screened via ELISA for high reactivity against Podxl isolated from human breast cancer MDA-MB-231 and low reactivity to the human embryonic kidney 293 (HEK 293) cells. Next, selected clones were screened against Podxl-expressing MDA-MB-231 and its PODXL-KO counterpart to confirm Podxl specificity. Supernatants were also screened for reactivity with Chinese hamster ovary (CHO) cells expressing Podxl, and low reactivity to CHO cells expressing related family members CD34 (Cd34) and endoglycan (Podxl2). Binding profiles for tumor versus normal cells were generated to enrich for those that preferentially bound to Podxl on tumor cells. Ab $\mathrm{V}_{\mathrm{H}}$ and $\mathrm{V}_{\mathrm{L}}$ regions were subsequently cloned into expression vectors containing the constant regions of the rabbit, human, or rabbit/human chimeric heavy and light chains, respectively, in order to generate high-affinity anti-Podxl rabbit, humanized and rabbit/human chimeric IgG I $_{1}$ Abs.

\section{Cell culture}

HEK293, human umbilical vein endothelial (HUVEC), SKOV3, SUM149, PANC-1, A-172, MIAPACA and MDAMB-231 cells were obtained from ATCC. OV3331 patient tumor-derived EOC cell line was kindly provided by Dr. Mes-Masson from the University of Montreal. HEK293, human pancreatic adenocarcinoma (PANC-1, MIAPACA), A-172 and MDA-MB-231 cells were grown in Dulbecco's Modified Eagle's Me (DMEM, Gibco, \#11965092) supplemented with $10 \%$ fetal bovine serum (FBS) and $10 \mathrm{U} / \mathrm{mL}$ penicillin and streptomycin $(\mathrm{P} / \mathrm{S})$ (Gibco, $\# 15140-122$ ). HUVEC cells were harvested from donor umbilical cords (Human Ethics no. H10-00643), grown in Endothelial Cell Growth Medium-2 Bulletkit (LONZA, \#CC-3162), and used between passages 2 and 8. Human ovarian cancer SKOV3 cells were grown in DMEM F-12 with $15 \mathrm{mM}$ 4-(2-hydroxyethyl)-1-piperazineethanesulfo nic acid (HEPES) (Sigma, \#D6421) supplemented with $10 \%$ FBS, $0.2 \mathrm{mM}$ L-glutamine (Gibco, \#25 030-081) and $10 \mathrm{U} / \mathrm{mL} \mathrm{P} / \mathrm{S}$. Human breast cancer SUM149 cells were grown in DMEM F-12 with $15 \mathrm{mM}$ HEPES supplemented with 5\% FBS, $5 \mu \mathrm{g} / \mathrm{mL}$ insulin (Sigma, \#234-291-2), $1 \mu \mathrm{g}$ / $\mathrm{mL}$ hydrocortisone (StemCell Technologies, \#07904) and $10 \mathrm{U} / \mathrm{mL} \mathrm{P} / \mathrm{S}$. OV3331 cells were grown in complete OSE medium. ${ }^{36}$ All cell lines were maintained at $37^{\circ} \mathrm{C}, 5 \% \mathrm{CO}_{2}$ and high humidity.

\section{CRISPR/Cas9 plasmid design}

To establish SKOV3, MIAPACA and MDA-MB-231 PODXL-KO cells, we followed a two-cut approach using clustered regularly interspaced short palindromic repeat 
(CRISPR)/CRISPR-associated nuclease 9 (Cas 9) technology. Target sites flanking the first exon were selected (combined cut size of $753 \mathrm{bp}$ ). Target sequences were designed using the Zhang's lab online CRISPR design tool (http://crispr.mit.edu). Oligonucleotides used to generate the guide RNAs (gRNAs) to edit the human PODXL gene were: g-PODXLup-FW (5'- caccgCCGCCCTGGAGCGCGACGGG-3'), g-PODXLup-RV (5'-aaac CCCGTCGCGCTCCAGGGCGGc-3'), g-PODXLdw-FW (5'-caccgGTGGAGACGAATCTACGCCC-3'), g-PODXLdw-RV (5' aaacGGGCGTAGATTCGTCTCCACc 3'). Each target's pair of annealed oligonucleotides was cloned into pSpCas9(BB)-2A-GFP (PX458) gRNA expression vector (Addgene \#48138; http://n2t.net/addgene: 48138; RRID:Addgene_48138) following digestion with BbsI (10 U/ $\mu$ l, ThermoFisher Scientific, \#ER1011) as previously described. ${ }^{37}$ These constructs were denominated pX458-Cas9-gPODXLup and pX458-Cas9-gPODXLdw, respectively. Sequences of all constructs were confirmed by Sanger sequencing.

\section{Generation of CRISPR/Cas9-mediated PODXL-KO cells}

SKOV3, MIAPACA and MDA-MB-231 cells $\left(5 \times 10^{5}\right)$ were co-transfected with $2 \mu \mathrm{g}$ of both pX458-Cas9-gPODXLup and pX458-Cas9-gPODXLdw using Lipofectamine 3000 Transfection Reagent (ThermoFisher Scientific, \#L3000015). Two days after transfection, cells were sorted for green fluorescent protein (GFP) expression. Three days post-sorting, cells were single-cell cloned in a 96-well plate. After 3-4 weeks, single colonies were divided in half; one half was used for propagation and colony maintenance while the other was used to assess the PODXL locus. A 983 bp fragment including the segment flanked by the gRNA target regions was PCR-amplified from genomic DNA using PODXL-CRISPR4-FW (5'-CTCCTCCCGAGTGGAGAGT-3') and PODXL-CRISPR4-RV (5'-CTCCGGATTTGCTCGTAGTG-3') primers. PCR products were verified using gel electrophoresis. Colonies with a $230 \mathrm{bp}$ amplicon were selected and PODXL expression was assessed using immunoblotting and flow cytometry.

\section{Flow cytometry}

Cells were washed $1 \times$ with $\mathrm{Ca}^{2+}$-free and $\mathrm{Mg}^{2+}$-free HBSS (Gibco, \#14170-112), incubated for $1-2 \mathrm{~min}$ at $37^{\circ} \mathrm{C}$ in a $0.25 \%$ trypsin solution, quenched with complete growth media, then centrifuged for $4 \mathrm{~min}$ at $394 \mathrm{~g}$, washed $2 \times$ with flow cytometry staining (FACS) buffer (PBS, $2 \mathrm{mM}$ EDTA, $5 \%$ FBS, $0.05 \%$ sodium azide) and transferred to a 96 well ' $v$ ' bottom plate. Cells were resuspended in $100 \mu \mathrm{L}$ blocking buffer (FACS buffer, $1 \mu \mathrm{g} / \mathrm{mL}$ of antiCD16/CD32 (clone 2.4G), 2\% rat serum) for $20 \mathrm{~min}$ at $4^{\circ} \mathrm{C}$ in the dark, then spun at $394 \mathrm{~g}$ for $4 \mathrm{~min}$ and incubated in $100 \mu \mathrm{L}$ primary $\mathrm{Ab}$ solution for $30 \mathrm{~min}$ at $4^{\circ} \mathrm{C}$ in the dark. Rabbit PODO83 ${ }^{21}(2 \mu \mathrm{g} / \mathrm{mL})$ and either rabbit or chimeric PODO447 $(5 \mu \mathrm{g} / \mathrm{mL})$ were used to detect Podxl. Rabbit IgG $(5 \mu \mathrm{g} / \mathrm{mL}$, Vector Laboratories, \#I-1000-5) and mouse/human-chimeric pavilizumab ( $5 \mu \mathrm{g} / \mathrm{mL}$, National Research Council (NRC) of Canada) were used as isotype controls. Next, cells were washed $3 \times$ with FACS buffer and resuspended in $100 \mu \mathrm{L}$ of secondary Ab solution (Alexa Fluor 647 (AF647) donkey-anti-rabbit $(2 \mu \mathrm{g} / \mathrm{mL}$, Invitrogen, \#A31573) or AF647 goat-antihuman $(2 \mu \mathrm{g} / \mathrm{mL}$, Jackson ImmunoResearch Laboratories, \#109-605-098)) for $30 \mathrm{~min}$ at $4^{\circ} \mathrm{C}$ in the dark. Cells were washed $2 \times$ with FACS buffer and resuspended in FACS buffer containing propidium iodide $(0.5 \mu \mathrm{g} / \mathrm{mL}$, Life Technologies, \#P3566). All flow cytometry data was acquired using a BD LSRII and analyzed using FlowJo software (BD Biosciences, Ashland).

\section{Glycan array}

Fabrication of the printed glycan array slides and highthroughput analysis were performed as previously described. ${ }^{38}$ Printed glycochips were incubated in blocking buffer $(50 \mathrm{mM}$ ethanolamine buffer, $\mathrm{pH} 8.5$ ) for 1 hour at room temperature (RT). Slides were rinsed with PBS $(3 \times, 30 \mathrm{~s})$, deionized water $(1 \times, 30 \mathrm{~s})$, and incubated with $50 \mu \mathrm{g} / \mathrm{mL}$ of humanized PODO447 or human IgG1א isotype control for 1 hour with gentle rotation (200 revolutions/min) in a sealed, humidified environment. Next, slides were washed with PBS-T (PBS, $0.05 \%$ Tween20, pH $7.4(1 \times, 5 \mathrm{~min}))$ and PBS $(1 \times, 30 \mathrm{~s})$, followed by a short centrifugation. Slides were incubated with goat antihuman IgG-Cy3 (Fc specific, $10 \mu \mathrm{g} / \mathrm{mL}$, SigmaAldrich, \#C2571) for 1 hour at RT. Slides were washed with PBS $(3 \times, 30 \mathrm{~s})$, deionized water $(1 \times, 30 \mathrm{~s})$, and dried by centrifugation $(30 \mathrm{~s})$. Fluorescent signals from the bound $\mathrm{Ab}$ were detected using ScanArray microarray scanner (PerkinElmer). Scanned images were analyzed using ScanArray Express software. Spots were identified using automated spotfinding with manual adjustments for occasional irregularities. Spot relative fluorescent (RFU) intensity was determined by subtracting the median pixel intensity of the local background from the average pixel intensity within the spot. Triplicate spots were averaged and the mean value of RFU intensity was used.

\section{Enzymatic treatment}

SKOV3 cells $\left(2.5 \times 10^{6}\right)$ were washed $1 \times$ with PBS, incubated in enzyme-free dissociation buffer (ThermoFisher Scientific, \#13151-014) for $15 \mathrm{~min}$, centrifuged at $394 \mathrm{~g}$ and washed $1 \times$ with assay buffer $\left(\mathrm{Ca}^{2+} / \mathrm{Mg}^{2+}\right.$ free DMEM/ F12 (Sigma, \#D9785), $2 \mathrm{mM} \mathrm{CaCl}_{2}, 0.1 \%$ BSA). Aliquots were resuspended into three treatment conditions: control $(500 \mu \mathrm{l}$; assay buffer), neuraminidase $(500 \mu \mathrm{L}$; $5 \mathrm{U} / \mathrm{mL}$ of neuraminidase (New England BioLabs, \#P0720S) in assay buffer), or endopeptidase treatment $(500 \mu \mathrm{L} ; 0.24 \mathrm{mg} / \mathrm{mL}$ of endopeptidase (Cedarlane, \#CLE100) in assay buffer). Cells were treated for $45 \mathrm{~min}$ at $37^{\circ} \mathrm{C}$, and tubes were inverted at $10 \mathrm{~min}$ intervals. Cells were then centrifuged at $394 g$ for 5 min and washed $2 \times$ with assay buffer prior to staining with rabbit PODO83, PODO447 and isotype control, and flow cytometric analysis. 


\section{Full-length and Podxl- $\Delta$ Mucin mutant-expressing SKOV3 epithelial OC cells}

Human full length (WT) and Podxl- $\Delta$ Mucin sequences were synthesized as gBlock gene fragments purchased from Integrated DNA Technologies, and assembled and cloned into the pTT22 expression vector (NRC of Canada) using Gibson assembly. ${ }^{39}$ Sequences were designed to include mouse CD43 signal peptide (SP) and a FLAG-tag motif (sequences in online supplemental table 1, online supplemental figure 1). Podxl- $\Delta$ Mucin mutant was generated by substituting Podxl's mucin domain for CD43's SP and FLAG-tag motif. Plasmids containing the constructs were confirmed by Sanger sequencing. SKOV3 $\left(5 \times 10^{5}\right)$ PODXL-KO cells were stably transfected with $2 \mu \mathrm{g}$ of either WT- or Podxl- $\Delta$ Mucin-pTT22 using Lipofectamine 3000 Transfection Reagent (ThermoFisher Scientific, \#L3000015), and maintained under puromycin selection (5 $\mu \mathrm{g} / \mathrm{mL}$, Gibco, \#A1113802). Expression was analyzed using western blotting and flow cytometry, and cells were sorted to enrich for construct-expressing cells.

\section{Immunohistochemistry}

OC tissue microarrays (TMA) were obtained from the Genetic Pathology Evaluation Centre TMA Database (Vancouver, Canada), and stained with either rabbitPODO83 $(2 \mu \mathrm{g} / \mathrm{mL})$ or rabbit-PODO447 $(5 \mu \mathrm{g} / \mathrm{mL})$. Tissue cross-reactivity (TCR) TMAs were purchased from US Biomax (\#MNO1021) and stained with either $\mathrm{Rbt} / \mathrm{Hu}$-chimeric PODO447-BIOT $(5 \mu \mathrm{g} / \mathrm{mL})$ or $\mathrm{Mo} /$ $\mathrm{Hu}$-chimeric palivizumab-BIOT control $(5 \mu \mathrm{g} / \mathrm{mL})$. Additionally, humanized PODO447 was sent to Charles Rivers Laboratories for independent testing of Ab TCR. See online supplemental materials and methods for a detailed staining protocol.

\section{Immunohistochemical scoring}

OC TMAs (219 cases) were blindly scored by four independent observers (DCH, KW, CDR), including a pathologist (MK), compared and combined. The TCR studies were performed by two independent facilities, including the preclinical contract research organization Charles River Laboratories (CRL) where the analysis was carried out by an independent pathologist under good laboratory practices. See online supplemental materials and methods for a detailed description of the scoring process.

\section{Generation of Ab-drug conjugates}

Monomethyl auristatin E (MMAE) linked to a synthetic dipeptide linker (MC-vc-PAB-MMAE) was used for conjugation with rabbit/human-chimeric PODO447 (NRC of Canada). Mouse/human-chimeric palivizumab was also conjugated with MMAE (MC-vc-PAB-MMAE) and served as the control (NRC). All conjugates were verified by high-performance liquid chromatography for the drug to Ab ratio (DAR) target of 4.0.

\section{Ab-drug conjugates in vitro cytotoxicity}

On day 0, OV3331, SKOV3 WT and PODXL-KO cells $\left(2.5 \times 10^{3}\right.$ per well $)$ were plated on 96 -well plates in $100 \mu \mathrm{L}$ culture medium and allowed to adhere overnight. On day 1 , a $5 \times$ stock solution of each mAb-antibody-drug conjugate (ADC) concentration to be tested was prepared in a stepwise 1:3 serial dilution series in cell culture medium, and $25 \mu \mathrm{L}$ of each dilution was added to cells in triplicate. Treated cells were cultured at $37^{\circ} \mathrm{C}, 5 \% \mathrm{CO}_{2}$ and high humidity for 144 hours. On day 6 , the thiazolyl blue tetrazolium bromide (MTT) assay was used to determine relative cytotoxicity. Briefly, $100 \mu \mathrm{L}$ of MTT $(1 \mathrm{mg} / \mathrm{mL}$, ThermoFischer Scientific, \#AC158990010) in culture medium was added to the cells and left to incubate for 3 hours at $37^{\circ} \mathrm{C}$. Next, MTT media was removed and $50 \mu \mathrm{L}$ of dimethyl sulfoxide (DMSO, Sigma, \#276855) was added to the cells and allowed to incubate for $15 \mathrm{~min}$ at RT, protected from light and with mild shaking. Absorbance was then read at $570 \mathrm{~nm}$. The per cent viability was calculated as follows: (1-(absorbance of treated samples/ average absorbance of control samples) $) \times 100$. Average relative cytotoxicity was calculated and statistical analysis was performed using two-way analysis of variance in GraphPad Prism software. A p value less than 0.05 was considered statistically significant.

\section{RESULTS \\ Development of a novel tumor-restricted anti-Podxl Ab}

We developed a panel of anti-Podxl mAbs that recognize the extracellular domain of Podxl using SLAM.$^{35}$ Briefly, rabbits were immunized with A-172 cells, a human glioblastoma cell line that expresses high levels of tumorglycosylated Podxl. Supernatants from splenic primary B cell clones were screened for reactivity to cell surfaceexpressed Podxl and minimal cross reactivity to Podxldeficient cells or cells expressing related family members CD34 and endoglycan.

Next, we screened Podxl-reactive B cell clones against Podxl-expressing human tumor cell lines from diverse tissue origins including SKOV3 (ovarian), A-172 (glioblastoma), MDA-MB-231 (breast) and MIAPACA (pancreatic) and counter-screened against normal HUVEC to identify tumor-specific Abs. We selected two mAbs for further characterization. The previously described PODOC1 (PODO83) ${ }^{21}$ showed high affinity for Podxl on both tumor and normal cells. Conversely, PODO447 showed high affinity only for Podxl expressed on tumor cell lines (figure 1A, online supplemental figure 2). To confirm Podxl-specificity of PODO447 and PODO83, we ablated Podxl expression in three PODO447/83-positive tumor lines (SKOV3, MIAPACA and MDA-MB-231) using CRISPR-mediated gene deletion. We observed complete loss of PODO447 and PODO83 binding on all CRISPRPODXL-knockout (PODXL-KO) cells compared with the parental (WT) lines (figure 1B), thus confirming that both PODO447 and PODO83's epitopes require expression of the Podxl protein.

To further assess the specificity of PODO447 for tumor Podxl, we evaluated the reactivity of PODO447 and PODO83 to normal human kidney, where Podxl is 


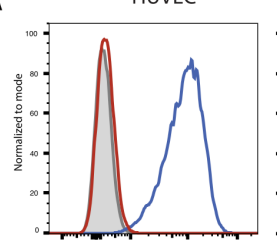

SUM 149

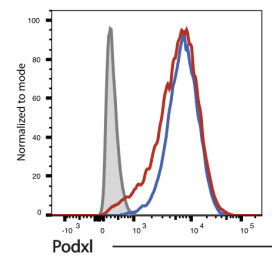

SKOV3
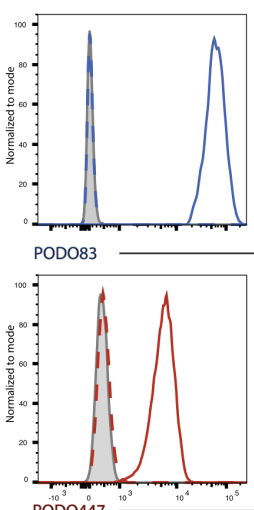

A-172

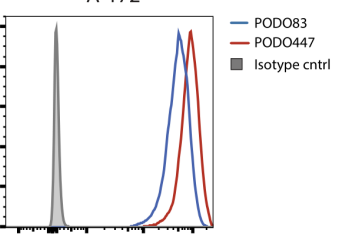

PANC-1

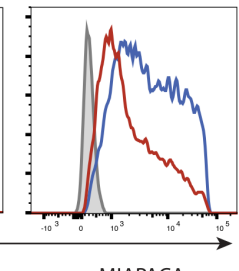

MIAPACA
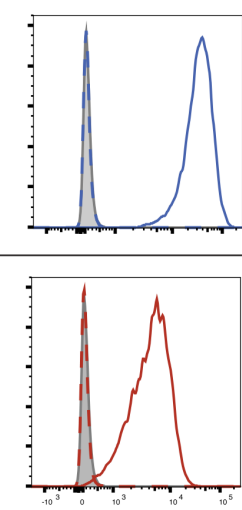
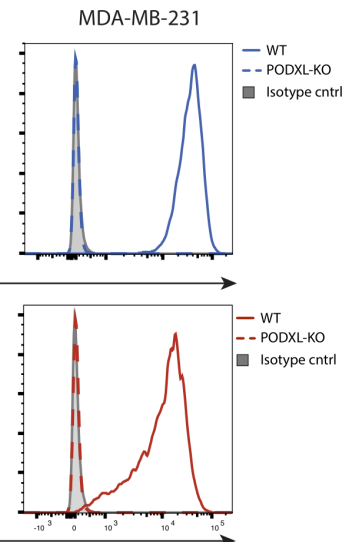

Figure 1 Development of novel antipodocalyxin antibodies. (A) Flow cytometric PODO83 (blue lines) and PODO447 (red lines) binding profiles of Podxl-positive normal (HUVEC) and tumor cells (A-172, SUM149, PANC-1). (B) Flow cytometric histograms showing PODO83 (blue lines) and PODO447 (red lines) immunoreactivity to Podxl-positive (WT, solid lines) or -deficient (PODXL-KO, dashed lines) ovarian (SKOV3), pancreatic (MIAPACA) and breast (MDA-MB-231) cancer cells. WT, wild type.

highly expressed by glomerular podocytes. ${ }^{15}$ Notably, in formalin-fixed, paraffin-embedded sections of the kidney cortex, PODO83 staining was readily evident on the glomerulus and vascular endothelium, whereas PODO447 staining was undetectable (figure 2A). Next, we evaluated PODO447's cross reactivity on an array of normal human histological sections. While PODO447 demonstrated intense staining on cryosections of control A-172 cells (online supplemental table 2), it was found to be minimally reactive on normal tissue (figure 2B, online supplemental table 3 ). We detected rare-tooccasional reactivity $(<10 \%)$ on mucosal epithelial cells in breast, fallopian tube and endometrium. In addition, we observed occasional weak cytosolic positivity in cells of the salivary gland and adrenal gland, stomach, rectum, pancreas and kidney cortex. This intracellular reactivity was also evident in tissues stained with an isotype-matched negative control, arguing against specific reactivity. Importantly, we found minimal expression of the PODO447 epitope on normal endothelia of all tissue sites examined. The sole exception was occasional vessel-like structures

A

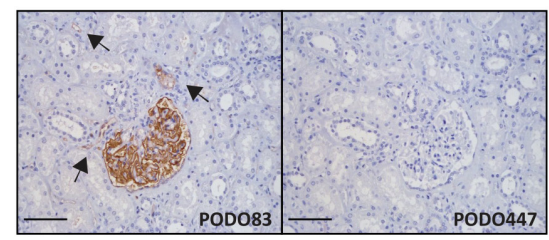

B

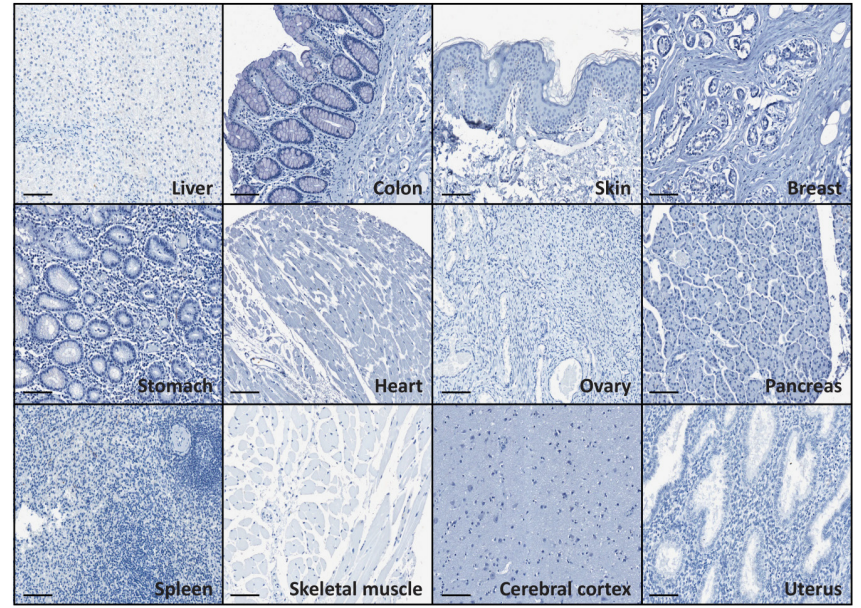

Figure 2 Representative images of healthy human tissue stained with PODO447. (A) Normal human kidney serial sections stained with either PODO83 or PODO447. Note the strong PODO83 staining (brown color) present on the podocytes in the glomerulus and on vascular endothelia (arrows). (B) Examples of normal human tissue sections stained with PODO447. Scale bars $=100 \mu \mathrm{m}$.

restricted to the kidney medulla that are likely vasa recta. In addition, PODO447's minimal normal TCR was independently confirmed by the CRL at both low $(2 \mu \mathrm{g} / \mathrm{mL})$ and high $(20 \mu \mathrm{g} / \mathrm{mL})$ Ab concentrations (online supplemental table 4). Concordantly, CRL found that PODO447 stained the cytoplasm of a very low number of podocytes, rare cells of the mucosal epithelia of the fallopian tube and small intestine, and rare cells of the skin sweat gland epithelia. Therefore, we conclude that PODO447 does not recognize the Podxl core protein (or other epitopes) expressed by most, if not all, normal tissue (figure 2B, online supplemental tables 3 and 4).

\section{P0D0447 binds selectively to the mucin domain of tumor- expressed PodxI}

Given that both PODO83 and PODO447 bind to the extracellular domain of the protein, we hypothesized that PODO447 may bind to a region subject to post-translational modifications and likely within the mucin domain. To test this, we performed a series of enzyme degradation assays using PODO83- and PODO447-reactive SKOV3 OC cells (figure 3A). Treatment of SKOV3 cells with neuraminidase, which removes terminal sialic acids on glycoproteins, did not affect binding of either Ab. However, O-sialoglycoprotein endopeptidase, a proteolytic enzyme that specifically cleaves proteins bearing clusters of negatively charged sugars, significantly attenuated PODO447, but not PODO83, immunoreactivity (figure 3A). From 
A

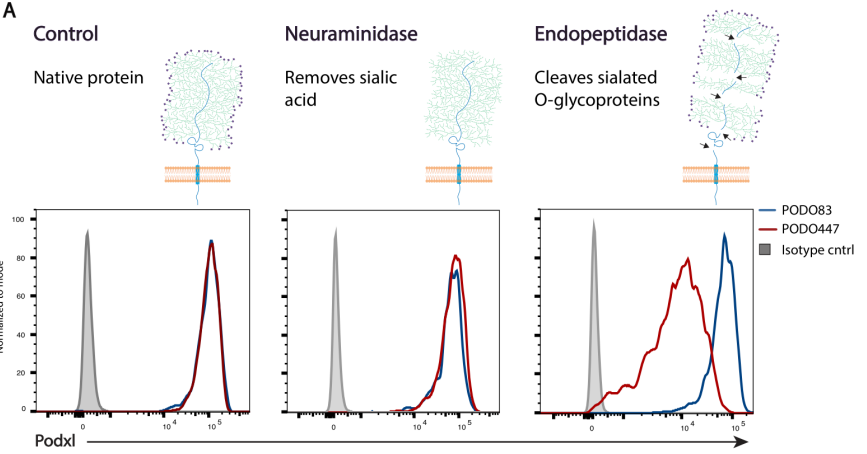

B

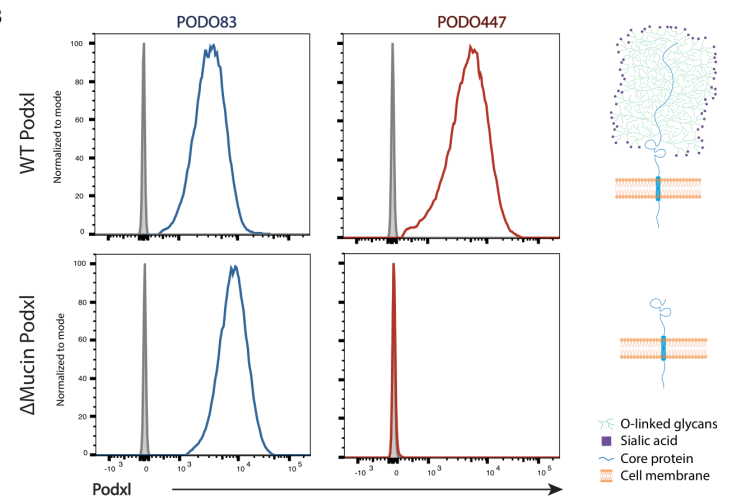

Figure 3 PODO447 recognizes a glycomotif on Podxl's mucin domain. (A) Flow cytometric histograms of SKOV3 wild-type (WT) cells treated with either assay buffer (control), neuraminidase (which removes the terminal sialic residues) or O-sialoglycoprotein endopeptidase (which cleaves the mucin domain). Cells were then stained with either PODO83 (blue) or PODO447 (red). (B) Flow cytometric histograms showing PODO83 (blue lines) and PODO447 (red lines) immunoreactivity to SKOV3 PODXL-KO cells re-expressing the WT podocalyxin (Podxl) (WT Podxl) protein or a Podxlmutant lacking the mucin domain ( $\triangle$ Mucin Podxl).

this, we conclude that the PODO447 epitope lies within the mucin domain.

To further confirm this result, we reconstituted SKOV3 PODXL-KO cells with either the full length, wild-type
(WT) protein or a mutant lacking the N-terminal mucin domain ( $\triangle$ Mucin), and evaluated the ability of these to restore the PODO83 and PODO447 epitope (figure 3B). Whereas both Abs bound to PODXL-KO cells expressing the WT protein, only PODO83 (but not PODO447) reacted with cells expressing the Podxl- $\Delta$ Mucin protein. From these data, we conclude that the PODO83 epitope is present within the extracellular stalk domain of Podxl, whereas PODO447 binds selectively to an epitope within the glycosylated mucin domain.

\section{P0D0447 recognizes a rare tumor-restricted glycoepitope}

Since PODO447 showed selective binding to the mucin domain of Podxl on tumor cells, we hypothesized that it may react with a modified glycoepitope generated exclusively by tumor cells. To gain further insights into its specificity, we probed a well-characterized glycan array ${ }^{40}$ with PODO447 to map potential glycoepitopes (table 1). This printed array includes 320 distinct glycan structures and serves as an ideal platform for identifying binding structures required for carbohydrate recognition. ${ }^{41}$ PODO447 exhibited the highest affinity to a rare, terminal N-acetylgalactosamine-beta-1 (GalNAc $\beta 1)$ motif on O-linked glycans (table 1 and online supplemental table $5)$. This glycomotif has been previously found on cancerassociated glycoepitopes such as globotetraose $(\mathrm{Gb} 4, \mathrm{P})^{42}$ and LadiNAc. ${ }^{43}$ In addition, PODO447 showed significant binding to the Tn antigen (GalNAcol-OSer), which has previously been reported to be abundantly expressed on Podxl in the kidney. ${ }^{44}$ However, the Tn antigen is most likely not involved in PODO447's recognition of cancer cells, as we observe a very low number of Tn-positive cells on cell lines expressing high levels of the PODO447 epitope (online supplemental figure 3). In addition, the anti-Tn $\mathrm{Ab}$, clone $5 \mathrm{~F} 4$, did not interfere with the ability of PODO447 to bind its epitope (online supplemental figure 4). To the best of our knowledge, this is the first time the GalNAc $\beta 1$ motif has been observed on Podxl, and PODO447 represents the first $\mathrm{mAb}$ against it.

Table 1 PODO447 recognizes a rare glycomotif

\begin{tabular}{|c|c|c|}
\hline Glycan structure & Common name & Median RFU $\left(10^{3}\right)$ \\
\hline GalNAc $\beta 1-3 G$ al $\alpha 1-4$ Gal $\beta 1-4 \mathrm{Glc} \beta-\mathrm{sp} 3$ & $\mathrm{~Gb} 4, \mathrm{P}$ & 46.9 \\
\hline GalNAc $\beta$-sp10 & beta-GalNAc & 37.8 \\
\hline GalNAc $\beta-s p 3$ & beta-GalNAc & 26.4 \\
\hline GaINAc $\alpha 1-O S e r$ & TnSer & 20.0 \\
\hline GalNAc $\alpha 1-3$ Gal $\beta$-sp3 & $A_{\mathrm{di}}$ & 5.7 \\
\hline Fuc $\alpha 1-2$ Gal $\beta$-sp3 & $\mathrm{H}_{\mathrm{di}}$ & 3.4 \\
\hline GalNAc $\beta 1-3$ GalNAc $\beta$-sp3 & para-Fs & 2.2 \\
\hline Fuc $\alpha 1-2(3-O-S u) G a l \beta-s p 3$ & $3-\mathrm{O}-\mathrm{Su}-\mathrm{H}_{\mathrm{di}}$ & 1.5 \\
\hline
\end{tabular}

List of top 10 glycans and magnitude of their median binding to PODO447. RFU=relative fluorescent units (range $0-50 \times 10^{3}$ ). See results of full array in online supplemental table 5 . 


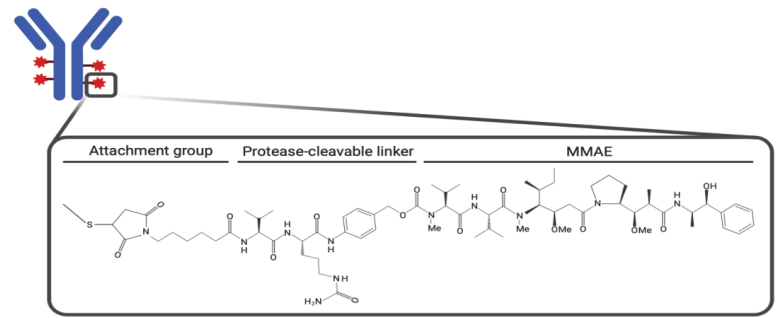

B

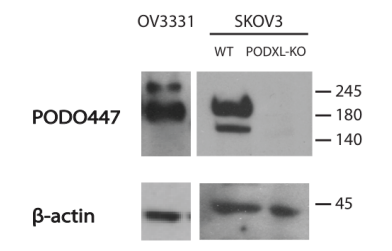

C

D
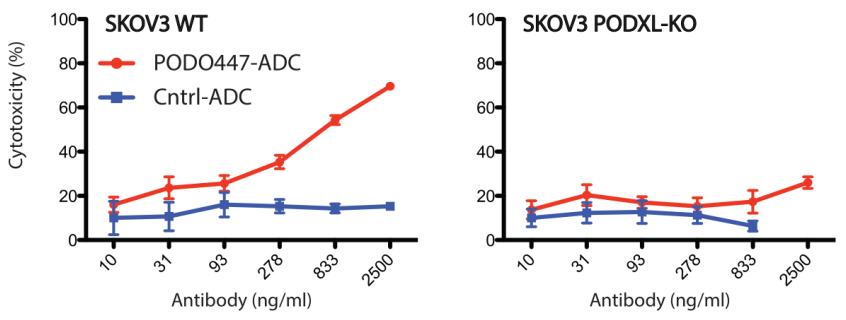

Figure 4 PODO447 antibody-drug conjugate in vitro cytotoxicity. (A) Representative image of mAb-Vedotin conjugate structure (created with BioRender.com). (B) Western blot showing PODO447 binding to lysates from OV3331, and SKOV3 WT and PODXL-KO cells. (C) In vitro cytotoxic effect of PODO447 and palivizumab-vedotin conjugates on OV3331 patient-derived ovarian high-grade serous cancer cells $(p<0.001)$. (D) In vitro cytotoxicity of control and PODO447-ADC on SKOV3 wild-type (WT) and $P O D X L-K O$ cells $(\mathrm{p}<0.001)$. Representative experiments are shown, and the values indicate the mean $\pm S D$. MMAE, monomethyl auristatin E.

\section{POD0447 drug conjugate results in tumor cytotoxicity}

The discovery that PODO447 recognizes a novel tumor epitope on Podxl encouraged us to investigate the potential use of PODO447 as a cancer therapeutic. We first assessed PODO447 as an unconjugated monotherapy and observed no effects of this $\mathrm{mAb}$ as a single agent in vitro or in vivo (online supplemental figure 5). These results encouraged us to investigate PODO447 as the targeting arm of an ADC immunotherapeutic. We chose MMAE as our toxin payload, a well-known tubulin polymerization inhibitor that has been used as a cancer therapy due to its potent antitumor activity. Next, we conjugated chimeric PODO447 and control palivizumab mAbs to MMAE using a valine-citrulline (val-cit) proteolytically cleavable linker, resulting in Vedotin (Val-Cit-MMAE) ADCs (figure 4A). The cytotoxic effects of both conjugates were determined in a long-term (144hours) drug exposure assay on OV3331, and SKOV3 WT and PODXL-KO cells (figure 4B-D). We observed a nine-fold reduction in cell viability in PODO447-ADC-treated OV3331 cells when compared to the control (figure 4C). This cytotoxic effect was due to specific drug delivery as we did not observe a reduction in cell viability on palivizumab-ADC-treated cells. To further confirm the specificity of PODO447 ADC to Podxl-expressing tumor cells, we treated SKOV3 WT and PODXL-KO with the vedotin conjugates. We observed a three-fold reduction in the viability of SKOV3 treated with PODO447-ADC but no effect on the PODXL-KO cells treated with PODO447-ADC (figure 4D). As expected, neither line responded to control-ADC-treatment (figure 4D). From these experiments, we conclude that PODO447 can effectively deliver, with precise specificity, a toxic payload to ovarian tumor cells expressing an appropriately tumor-glycosylated form of Podxl.

\section{P0D0447 as a therapeutic candidate for OC}

The finding that PODO447 recognizes a highly unusual tumor-restricted residue present on Podxl that does not crossreact with normal human tissues and could be used to deliver a toxic payload prompted us to further evaluate possible PODO447 therapeutic indications. The Podxl core protein is known to be expressed at high levels in $~ 90 \%$ of tuboovarian high-grade serous ovarian carcinomas (HGSOC), where cell surface localization is a strong predictor of poor outcome. $^{22}$ Given the high lethality of HGSOC tumors, we carried out a detailed evaluation of the PODO447 epitope expression by these malignancies in an effort to evaluate the potential of PODO447 as a new immunotherapy for this cancer. We immunostained a TMA consisting of 219 cases of OC with PODO447 (TMA clinicopathological characteristics in online supplemental table 6). Each case on the TMA was initially scored based on the percentage of cells stained, with a scoring scale of 0 (negative, $<1 \%$ ), +1 (intermediate, $1 \%-50 \%$ ) and +2 (high, $>50 \%$; table 2 ). Tumors scored as +1 and +2 were grouped as PODO 447 positive. Of 219 cases, $144(65.8 \%)$ were PODO447 positive. Positivity was not correlated with either the International Federation of Obstetrics and Gynecology stage or disease-free survival across the entire OC cohort (table 2, data not shown).

We did note, however, that PODO447 reactivity across the major OC histotypes was significantly different. While only $40 \%$ of the mucinous carcinomas were PODO447 positive, the majority of endometrioid $(74.1 \%)$, clear cell $(68.0 \%)$, low-grade serous (75.0\%) and HGSOC (65.8\%) were PODO447 positive.

We have previously shown that cell surface localization of Podxl (vs cytoplasmic localization) is associated with decreased disease-free survival in HGSOC. ${ }^{22}$ Accordingly, we also scored PODO447's predominant localization (cytoplasmic vs membranous) among the PODO447-positive cases on the TMA (table 2, figure 5). Overall, $115(79.9 \%)$ of the 144 PODO447-positive cases exhibited membranous PODO447 staining (table 2, figure 5B). The remaining 29 cases exhibited diffuse, cytoplasmic staining (table 2, figure 5C). Unfortunately, due to the lower representation of mucinous and low-grade serous tumors in the TMA, we were unable to infer any meaningful prognostic conclusions about PODO447 localization in these histotypes. However, 
Table 2 PODO447 expression and localization within the tissue microarray cohort

\begin{tabular}{lllll}
\hline & & \multicolumn{2}{c}{ PODO447 positive } \\
\cline { 3 - 5 } Patient characteristics & $\begin{array}{l}\text { Scorable } \\
\text { cases } \mathbf{n}\end{array}$ & $\begin{array}{l}\text { Total positive } \\
\mathbf{n}(\%)\end{array}$ & $\begin{array}{l}\text { Cell surface positive } \\
\mathbf{n}(\%)\end{array}$ & $\begin{array}{l}\text { Cytoplasmic positive } \\
\mathbf{n}(\%)\end{array}$ \\
\hline All scorable cases & 219 & $144(65.8)$ & $115(79.9)$ & $29(20.2)$ \\
\hline Histological cell type $(\mathrm{n})$ & & & $78(76.5)$ & $24(23.5)$ \\
\hline High-grade serous & 158 & $102(64.6)$ & $14(82.4)$ & $3(17.7)$ \\
\hline Clear cell & 25 & $17(68.0)$ & $19(95.0)$ & $1(5.0)$ \\
\hline Endometroid & 27 & $20(74.1)$ & $1(50.0)$ & $1(50.0)$ \\
\hline Mucinous & 5 & $2(40.0)$ & $3(100.0)$ & $0(0.0)$ \\
\hline Low-grade serous & 4 & $3(75.0)$ & & $6(20.7)$ \\
\hline FIGO stage (n) & & & $23(79.3)$ & $2(10.0)$ \\
\hline I & 37 & $29(78.4)$ & $18(90.0)$ & $17(20.5)$ \\
\hline II & 31 & $20(64.5)$ & $66(79.5)$ & $4(33.3)$ \\
\hline III & 130 & $83(63.8)$ & $8(66.7)$ & \\
\hline IV & 21 & $12(57.1)$ & &
\end{tabular}

FIGO, International Federation of Obstetrics and Gynecology.
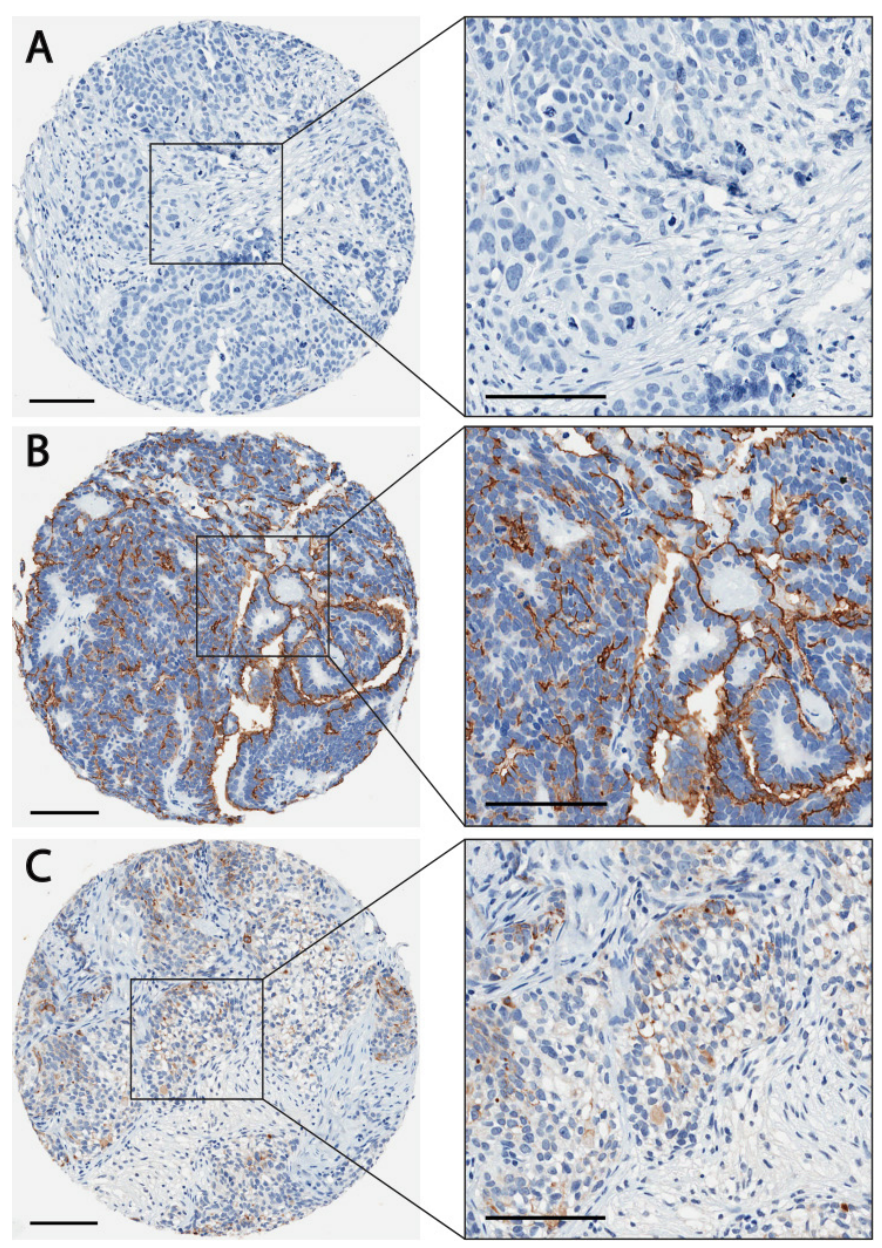

Figure 5 PODO447 differentially recognizes high-grade serous ovarian carcinomas (HGSOC) in a tumor microarray. Representative HGSOC tumor sections stained with PODO447 (brown color) that were scored as (A) negative (B) positive (membranous staining) or (C) positive (cytoplasmic) (see inset for higher magnification). Scale bars $=100 \mu \mathrm{m}$.
19 of $20(95.0 \%)$ endometrioid, 14 of $17(82.4 \%)$ clear cell and 78 of $102(76.5 \%)$ HGSOC tumors exhibited cell-surface PODO447-positive staining. Given that HGSOC is the most prevalent and lethal OC histotype, ${ }^{25}{ }^{46}$ the cell surface reactivity of PODO447 with the majority of these tumors bodes well for this mAb's utility in targeting and treating this unmet clinical need.

\section{DISCUSSION}

Podxl is a member of the CD34 family of sialomucins, a family of transmembrane proteins that has been shown to play a role in modifying cell adhesion and making cells more mobile and invasive. ${ }^{47}$ Interestingly, Podxl is frequently upregulated in a variety of cancers and invariably correlates with poor outcome. ${ }^{20-30}$ In those studies where its oncogenic function has been evaluated in detail, Podxl has been shown to play a critical role in metastatic progression; gene inactivation or dampening of its mRNA expression cripples the ability of tumor cells to metastasize in xenografted mice. ${ }^{20} 2932$ Thus, as a membrane protein involved in tumor progression, together with our previous study showing that Abs targeting its extracellular domain can delay primary tumor growth and block metastasis in mice, ${ }^{21}$ Podxl stands out as a promising target for immunotherapy. Further development of a Podxltargeted therapeutic $\mathrm{Ab}$ has been hampered, however, by concerns over potential toxicity since Podxl is also normally expressed at high levels by vascular endothelia and kidney podocytes. Accordingly, in this study we sought to screen for a novel mAb with high specificity for a tumor glycoform of Podxl, and lack of reactivity with any normal cells. PODO447 emerged as uniquely fulfilling these key criteria. This $\mathrm{mAb}$ recognizes an array of human tumor cell lines while exhibiting minimal activity with normal cell types or tissues, including those that express the Podxl core protein at high levels. Specifically, we observed no reactivity of PODO447 
with kidney podocytes and, with the sole notable exception of vessel-like structures in kidney medulla that appear to be vasa recta (under investigation), we observed minimal binding of PODO447 to any other peripheral vessels. Although we observed rare and weak PODO447 reactivity with the mucosal epithelia of the breast, fallopian tube and intestinal track, as well as the parenchyma of the adrenal, salivary and sweat glands, the level and frequency of this reactivity was low and could, in fact, represent spurious cross-reactivity since, in most cases, a similar staining pattern was also observed in the staining control. Moreover, the rare staining of these normal epithelial cells was observed to be largely cytoplasmic making it unlikely that this compartment would be accessible to circulating PODO447 in vivo. Importantly, these results have been independently confirmed by a preclinical CRO which speaks to the reproducibility and reliability of our findings.

With regards to therapeutic use of PODO447-based agents, epithelial ovarian cancer has emerged as an extremely promising avenue of further exploration. The Podxl core protein is known to be expressed at robust levels by the majority of high-grade serous carcinomas, ${ }^{22}$ the most prevalent histotype of $\mathrm{OC}^{49}$ for which the prognosis is poor and antigen-specific therapeutic options are extremely limited. ${ }^{45}{ }^{46}$ We found that a high percentage of such HGSOC tumors express the PODO447 glycoepitope, thus offering a large population who could benefit from treatment. As shown in this study, coupling of PODO447 with various cytotoxic compounds to selectively target neoplastic cells could offer a novel therapeutic approach with minimal toxicity to normal tissues, an issue that plagues most traditional chemotherapy treatments.

Although we have explored the reactivity of PODO447 in OC in detail, further studies on other forms of cancer could widen the scope of its potential therapeutic use. For example, Podxl is known to be expressed by a high percentage of colon, brain and pancreatic tumors and thus, provided they share the appropriate post-translation modification, they too could be promising avenues for targeted therapeutic intervention using this $\mathrm{Ab}$. As with any therapy, a nagging concern is the ability of cells to become resistant through down-regulation of the target antigen. However, because we have previously shown that Podxl plays a direct functional role in endowing metastatic cells with rare tumor initiating capacity, resistance may be less of an issue for this target. ${ }^{21}{ }^{32}$ Indeed, one could speculate that targeting the PODO447-expressing fraction of tumor cells may selectively eliminate the subset of cells with the highest metastatic capacity, making it a more targeted therapy for this elusive subset of cells than standard chemotherapy (currently under investigation).

Another potential concern for PODO447, in particular, is the emergence of cells that have lost the glycotransferases required to generate the PODO447 epitope. Yet here, too, the ability to become resistant will depend on the functional significance of the unusual glycosylation on the PODO447 epitope. The GalNAc $\beta 1$ glycomotif that comprises part of this epitope is extremely unusual in normal eukaryotic settings. However, similar glycan structures such as the LacdiNActype (GalNAcß1-4GlcNAc) motifs and the $\mathrm{P}$ antigen (Gb4) have previously been identified in ovarian cancer tissue. ${ }^{43}$
Thus, these epitopes may have an important functional role in tumor cell spread. If this were indeed the case, one could envision that targeting a rare PODO447 subpopulation of tumor cells would have an outsized role in managing the cancers by targeting cells most likely to spread. To this end, we are currently working to test the therapeutic efficacy of PODO447 coupled to toxins and radioisotopes in xenograft mouse tumor models.

Finally, the PODO447 epitope could offer promise as a new target for chimeric antigen receptor $\mathrm{T}$ cell (CAR-T)based therapies. Although CAR-T therapy has been transformative for hard-to-treat B cell malignancies (B cell leukemia and some B cell lymphomas), to date this approach has not been exploited widely for other malignancies and, in particular, for solid tumors. To a large extent, this is due to the lack of appropriate tumor-specific epitopes. In this regard, it is noteworthy that the success of CD19-specific CAR-T therapies for B cell malignancies largely reflects the ability of patients to survive the complete ablation of both the tumor and the normal Ab producing $\mathrm{B}$ cell compartment. From this vantage point, $\mathrm{PODO} 447$, as a highly tumor-restricted $\mathrm{mAb}$, may offer an unprecedented opportunity to extend the promise of this approach to ovarian cancers and other tumors.

\section{CONCLUSIONS}

PODO447 is a novel Podxl-targeting mAb that binds to a unique tumor-restricted glycoepitope with high specificity. Unlike other anti-Podxl Abs, PODO447 does not recognize Podxl expressed on healthy human tissue. Therefore, due to its potentially favorable safety profile, this study supports further development of PODO447 as a therapeutic agent.

\section{Author affiliations}

${ }^{1}$ The Biomedical Research Centre and School of Biomedical Engineering, The University of British Columbia, Vancouver, British Columbia, Canada

${ }^{2}$ Department of Cellular and Physiological Sciences, The University of British Columbia, Vancouver, British Columbia, Canada

${ }^{3}$ Centre for Drug Research and Development, Vancouver, British Columbia, Canada ${ }^{4}$ Copenhagen Center for Glycomics and Department of Cellular and Molecular Medicine (ICMM), University of Copenhagen, Kobenhavn, Denmark

${ }^{5}$ Department of Pathology and Laboratory Medicine, University of Calgary, Calgary, Alberta, Canada

${ }^{6}$ Department of Pathology and Laboratory Medicine, The University of British Columbia, Vancouver, British Columbia, Canada

Acknowledgements The authors are very grateful to Anna von Rossum, Patrick Chan, Bradley Hedberg, John Babcook, and all the members of the Biologics Division at CDRD for the development and validation of the Abs; to Tate Goodman and Alannah Wilson for their technical contributions characterizing the PODXL-KO cell lines; to Anne-Marie Mes-Masson for kindly providing the patient-derived OV3331 cell line; to David Huntsman, Samuel Leung and Ingrid Barta for providing expert technical advice; and to the Biomedical Research Center core members R. Dhesi (safety), M. Williams and B. Silva (Abs). They thank Alissa Cait, Jessica Cait and Christine Eisner for their critical reading of the manuscript.

Contributors DCH designed and performed most experiments, analyzed and interpreted data, and was the primary author of this manuscript. MRH assisted with experimental design and manuscript writing. PD created the SKOV3 and MIAPACA PODXL-KO cell lines, initiated the enzymatic treatment experiments, and assisted in flow cytometry assays. PB, IS, CBG and EC assisted with the characterization of the antibodies studied herein. $\mathrm{OB}$ performed the glycan array experiments and analysis. MK and KW assisted with the ovarian carcinoma TMA immunohistochemical scoring, YL assisted in flow cytometry assays and CBG contributed the ovarian 
TMAs. CR and KM were key contributors to the experimental concept and design. All authors approved the final manuscript.

Funding This research was supported by the Canadian Institutes of Health Research (Grant Number: PJT-166180), the Canadian Cancer Research Institute (Grant number: 704344), the Center for Blood Research Graduate Award Program, Impact Grant from the Stem Cell Network Center of Excellence and the Danish National Research Foundation (Grant number: DNRF107).

Competing interests The authors are inventors of a pending patent application on P0D0447 and methods of using the same (US20180296673A1). MRH, PB, IS, CBG, $\mathrm{KM}$ and $\mathrm{CR}$ are inventors of a pending patent application on POD083 and methods of using the same (US20190367606A1). KM and CR possess an awarded patent on podocalyxin as a prognostic marker in cancer (US9309323B2).

\section{Patient consent for publication Not required}

Ethics approval This study was performed in consideration with the Tri-Council Policy Statement of Ethical Conduct for Research involving Humans (TCPS 2). Animal experiments were performed humanely based on recommendations of the Canadian Committee on Animal Care with approval of the University of British Columbia's Animal Care Committee (A16-0007, to KM).

Provenance and peer review Not commissioned; externally peer reviewed.

Data availability statement Data are available upon reasonable request. All data relevant to the study are included in the article or uploaded as supplementary information. All data supporting the results presented in this study is included and discussed in the article and its supplementary figures. Additional data not directly relevant for the study presented here will be made available upon reasonable request to the corresponding author.

Supplemental material This content has been supplied by the author(s). It has not been vetted by BMJ Publishing Group Limited (BMJ) and may not have been peer-reviewed. Any opinions or recommendations discussed are solely those of the author(s) and are not endorsed by BMJ. BMJ disclaims all liability and responsibility arising from any reliance placed on the content. Where the content includes any translated material, BMJ does not warrant the accuracy and reliability of the translations (including but not limited to local regulations, clinical guidelines, terminology, drug names and drug dosages), and is not responsible for any error and/or omissions arising from translation and adaptation or otherwise.

Open access This is an open access article distributed in accordance with the Creative Commons Attribution Non Commercial (CC BY-NC 4.0) license, which permits others to distribute, remix, adapt, build upon this work non-commercially, and license their derivative works on different terms, provided the original work is properly cited, appropriate credit is given, any changes made indicated, and the use is non-commercial. See http://creativecommons.org/licenses/by-nc/4.0/.

\section{ORCID iDs}

Diana Canals Hernaez http://orcid.org/0000-0003-4422-2839

Kelly M McNagny http://orcid.org/0000-0003-4737-3499

\section{REFERENCES}

1 Bray F, Ferlay J, Soerjomataram I, et al. Global cancer statistics 2018: GLOBOCAN estimates of incidence and mortality worldwide for 36 cancers in 185 countries. CA Cancer J Clin 2018;68:394-424.

2 National Cancer Institute. SEER - cancer of the ovary [Internet] Available: https://seer.cancer.gov/statfacts/html/ovary.html [Accessed 21 Nov 2019].

3 Vaughan S, Coward JI, Bast RC, et al. Rethinking ovarian cancer: recommendations for improving outcomes. Nat Rev Cancer 2011;11:719-25.

4 Torre LA, Trabert B, DeSantis CE, et al. Ovarian cancer statistics, 2018. CA Cancer J Clin 2018;68:284-96.

5 Fuster MM, Esko JD. The sweet and sour of cancer: glycans as novel therapeutic targets. Nat Rev Cancer 2005;5:526-42.

6 Peixoto A, Relvas-Santos M, Azevedo R, et al. Protein glycosylation and tumor microenvironment alterations driving cancer hallmarks. Front Oncol 2019;9:8256.

7 Shental-Bechor D, Levy Y. Effect of glycosylation on protein folding: a close look at thermodynamic stabilization. Proc Natl Acad Sci U S A 2008;105:8256-61.

8 Vagin O, Kraut JA, Sachs G. Role of N-glycosylation in trafficking of apical membrane proteins in epithelia. Am J Physiol Renal Physiol 2009;296:F459-69.

9 Ohtsubo K, Marth JD. Glycosylation in cellular mechanisms of health and disease. Cell 2006;126:855-67.
10 Stowell SR, Ju T, Cummings RD. Protein glycosylation in cancer. Annu Rev Pathol 2015;10:473-510.

11 Ono M, Hakomori S. Glycosylation defining cancer cell motility and invasiveness. Glycoconj J 2004;20:71-8.

12 Lima L, Neves M, Oliveira MI, et al. Sialyl-Tn identifies muscleinvasive bladder cancer basal and luminal subtypes facing decreased survival, being expressed by circulating tumor cells and metastases. Urol Oncol 2017;35:675.e1-675.e8.

13 Schultz MJ, Swindall AF, Bellis SL. Regulation of the metastatic cell phenotype by sialylated glycans. Cancer Metastasis Rev 2012;31:501-18.

14 Sassetti C, Van Zante A, Rosen SD. Identification of endoglycan, a member of the CD34/podocalyxin family of sialomucins. J Biol Chem 2000;275:9001-10.

15 Nielsen JS, McNagny KM. The role of podocalyxin in health and disease. J Am Soc Nephrol 2009;20:1669-76.

16 Doyonnas R, Kershaw DB, Duhme C, et al. Anuria, omphalocele, and perinatal lethality in mice lacking the CD34-related protein podocalyxin. J Exp Med 2001;194:13-28.

17 Doyonnas R, Nielsen JS, Chelliah S, et al. Podocalyxin is a CD34related marker of murine hematopoietic stem cells and embryonic erythroid cells. Blood 2005;105:4170-8.

18 Horrillo A, Porras G, Ayuso MS, et al. Loss of endothelial barrier integrity in mice with conditional ablation of podocalyxin (Podxl) in endothelial cells. Eur J Cell Biol 2016;95:265-76.

19 Cait J, Hughes MR, Zeglinski MR, et al. Podocalyxin is required for maintaining blood-brain barrier function during acute inflammation. Proc Natl Acad Sci U S A 2019:116:4518-27.

20 Somasiri A, Nielsen JS, Makretsov N, et al. Overexpression of the anti-adhesin podocalyxin is an independent predictor of breast cancer progression. Cancer Res 2004;64:5068-73.

21 Snyder KA, Hughes MR, Hedberg B, et al. Podocalyxin enhances breast tumor growth and metastasis and is a target for monoclonal antibody therapy. Breast Cancer Res 2015;17:46.

22 Cipollone JA, Graves ML, Köbel M, et al. The anti-adhesive mucin podocalyxin may help initiate the transperitoneal metastasis of high grade serous ovarian carcinoma. Clin Exp Metastasis 2012;29:239-52.

23 Itai S, Yamada S, Kaneko MK, et al. Immunohistochemical analysis using Antipodocalyxin monoclonal antibody PcMab-47 demonstrates podocalyxin expression in oral squamous cell carcinomas. Monoclon Antib Immunodiagn Immunother 2017;36:220-3.

24 Dallas MR, Chen S-H, Streppel MM, et al. Sialofucosylated podocalyxin is a functional E- and L-selectin ligand expressed by metastatic pancreatic cancer cells. Am J Physiol Cell Physiol 2012;303:C616-24.

25 Hsu Y-H, Lin W-L, Hou Y-T, et al. Podocalyxin EBP50 ezrin molecular complex enhances the metastatic potential of renal cell carcinoma through recruiting Rac1 guanine nucleotide exchange factor ARHGEF7. Am J Pathol 2010;176:3050-61.

26 Wu H, Yang L, Liao D, et al. Podocalyxin regulates astrocytoma cell invasion and survival against temozolomide. Exp Ther Med 2013;5:1025-9.

27 Favreau AJ, Cross EL, Sathyanarayana P. miR-199b-5p directly targets PODXL and DDR1 and decreased levels of miR-199b-5p correlate with elevated expressions of PODXL and DDR1 in acute myeloid leukemia. Am J Hematol 2012;87:442-6.

28 Zhou Y, Zhang L, Pan $\mathrm{H}$, et al. Bmi1 essentially mediates podocalyxin-enhanced cisplatin chemoresistance in oral tongue squamous cell carcinoma. PLoS One 2015;10:e0123208.

29 Wang J, Zhao Y, Qi R, et al. Prognostic role of podocalyxin-like protein expression in various cancers: a systematic review and metaanalysis. Oncotarget 2017;8:52457-64.

30 McNagny KM, Hughes MR, Graves ML, et al. Podocalyxin in the Diagnosis and Treatment of Cancer. In: Mohan PR, ed. Podocalyxin in the diagnosis and treatment of cancer. InTech KW, 2012.

31 Wong BS, Shea DJ, Mistriotis P, et al. A direct PodocalyxinDynamin-2 interaction regulates cytoskeletal dynamics to promote migration and metastasis in pancreatic cancer cells. Cancer Res 2019;79:2878-91.

32 Fröse J, Chen MB, Hebron KE, et al. Epithelial-Mesenchymal transition induces podocalyxin to promote extravasation via ezrin signaling. Cell Rep 2018;24:962-72.

33 Pinho SS, Reis CA. Glycosylation in cancer: mechanisms and clinica implications. Nat Rev Cancer 2015;15:540-55.

34 Pearce OMT, Läubli $\mathrm{H}$. Sialic acids in cancer biology and immunity. Glycobiology 2016;26:111-28.

35 Babcook JS, Leslie KB, Olsen OA, et al. A novel strategy for generating monoclonal antibodies from single, isolated lymphocytes producing antibodies of defined specificities. Proc Natl Acad Sci U S A 1996;93:7843-8. 
36 Sauriol A, Simeone K, Portelance L, et al. Modeling the diversity of epithelial ovarian cancer through ten novel well characterized cell lines covering multiple subtypes of the disease. Cancers 2020;12:2222.

37 Ran FA, Hsu PD, Wright J, et al. Genome engineering using the CRISPR-Cas9 system. Nat Protoc 2013;8:2281-308.

38 Huflejt ME, Vuskovic M, Vasiliu D, et al. Anti-Carbohydrate antibodies of normal sera: findings, surprises and challenges. Mol Immunol 2009;46:3037-49.

39 Gibson DG, Young L, Chuang R-Y, et al. Enzymatic assembly of DNA molecules up to several hundred kilobases. Nat Methods 2009;6:343-5.

40 Bovin N, Obukhova P, Shilova N, et al. Repertoire of human natural anti-glycan immunoglobulins. do we have auto-antibodies? Biochim Biophys Acta 2012;1820:1373-82.

41 Blixt O, Head S, Mondala T, et al. Printed covalent glycan array for ligand profiling of diverse glycan binding proteins. Proc Natl Acad Sci U S A 2004;101:17033-8.

42 Jacob F, Anugraham M, Pochechueva T, et al. The glycosphingolipid $\mathrm{P}_{1}$ is an ovarian cancer-associated carbohydrate antigen involved in migration. Br J Cancer 2014;111:1634-45.
43 Anugraham M, Jacob F, Everest-Dass AV, et al. Tissue glycomics distinguish tumour sites in women with advanced serous adenocarcinoma. Mol Oncol 2017;11:1595-615.

44 Stotter BR, Talbot BE, Capen DE, et al. Cosmc-dependent mucintype $O$-linked glycosylation is essential for podocyte function. Am J Physiol Renal Physiol 2020;318:F518-30.

45 Gadducci A, Guarneri V, Peccatori FA, et al. Current strategies for the targeted treatment of high-grade serous epithelial ovarian cancer and relevance of BRCA mutational status. J Ovarian Res 2019;12:1-8.

46 Lisio M-A, Fu L, Goyeneche A, et al. High-grade serous ovarian cancer: basic sciences, clinical and therapeutic Standpoints. Int J Mol Sci 2019;20:952.

47 Nielsen JS, McNagny KM. Novel functions of the CD34 family. J Cell Sci 2008;121:3683-92.

48 Graves ML, Cipollone JA, Austin P, et al. The cell surface mucin podocalyxin regulates collective breast tumor budding. Breast Cancer Res 2016;18:11.

49 Kurman RJ, Shih I-M. Molecular pathogenesis and extraovarian origin of epithelial ovarian cancer--shifting the paradigm. Hum Patho 2011;42:918-31. 